\title{
Predictive Value of Post-Transplant Bone Marrow Plasma Cell Percent in Multiple Myeloma Patients Undergone Autologous Transplantation
}

\author{
In Hye Hwang, Joo Seop Chung, Ho Jin Shin, Young Jin Choi, Moo Kon Song, Young Mi Seol, Goon Jae Cho, \\ Bo Gwang Choi, Mun Ki Choi, Bo Kyung Choi, Kang Hee Ahn, Kyung Hwa Shin, Hee Sun Lee, Hyung Seok Nam, \\ and Jong Min Hwang
}

Department of Internal Medicine, School of Medicine, Pusan National University, Busan, Korea

Background/Aims: Autologous stem cell transplantation (ASCT) has become the treatment of choice for patients with multiple myeloma (MM). Studies have shown that maintenance treatment with interferon-alpha is associated with improved survival rates following ASCT. However, despite these recent advances in regimes, relapses are inevitable; thus, the prediction of relapse following ASCT requires assessment.

Methods: We retrospectively analyzed 39 patients who received ASCT between 2003 and 2008. All patients received chemotherapy with vincristine, adriamycin, and dexamethasone (VAD), and ASCT was performed following high-dose melphalan conditioning therapy. We evaluated the influence of the post-transplant day $+14(D+14)$ bone marrow plasma cell percent (BMPCp) $(\geq 2$ vs. $<2 \%$ ), international scoring system (ISS) stage (II vs. III), response after 3 cycles of VAD therapy (complete response [CR] vs. non-CR), deletion of chromosome 13q (del[13q]) (presence of the abnormality vs. absence), and BMPCp at diagnosis ( $\geq 50 \mathrm{vs.}<50 \%$ ) on progressionfree survival (PFS) and overall survival (OS).

Results: During the median follow-up of 28.0 months, the median PFS and OS were 29.1 and 42.1 months, respectively. By univariate analysis, ISS stage III at diagnosis, BMPCp $\geq 50 \%$ at diagnosis, CR after 3 cycles of VAD therapy, del (13q) by fluorescence in situ hybridization, and BMPC $p \geq 2 \%$ at post-transplant $D+14$ were correlated with PFS and OS. A multivariate analysis revealed that a post-transplant $D+14$ BMPCp $\geq 2 \%$ (PFS, hazard ratio $[\mathrm{HR}]=4.426, p=0.008$; OS, $\mathrm{HR}=3.545, p=0.038$ ) and $\mathrm{CR}$ after 3 cycles of VAD therapy (PFS, HR = $0.072, p=0.014$; OS, $\mathrm{HR}=0.055, p=0.015$ ) were independent prognostic parameters.

Conclusions: Post-transplant D+14 BMPCp is a useful parameter for predicting the outcome for patients with MM receiving ASCT. (Korean J Intern Med 2011;26:76-81)

Keywords: Multiple myeloma; Stem cell transplantation; Bone marrow; Plasma cell

\section{INTRODUCTION}

Multiple myeloma (MM) is an incurable disease with a median survival of three years after conventional chemotherapy. High-dose chemotherapy followed by autologous stem cell transplantation (ASCT) is currently the standard treatment for patients with MM aged below
65 years. Several studies have shown that high-dose ASCT is associated with an improved response rate and prolonged progression-free survival (PFS) and overall survival (OS) compared to conventional chemotherapy $[1,2]$. In a recent study, alpha-interferon maintenance treatment was shown to be associated with improved survival rates after high-dose treatment and ASCT in patients with MM [3]. Unfortunately, despite these recent advances,

Received: July 1, 2010

Revised : August 30, 2010

Accepted: October 25, 2010 
relapses are frequent; thus, the development of effective diagnostic approaches that can anticipate the response duration of ASCT-based strategies is required.

Several prognostic factors following ASCT, including cytogenetic abnormalities (e.g., the deletion of chromosome 13q [del(13q)]), the plasma cell labeling index (PCLI), and the international scoring system (ISS), have been reported to be useful parameters of patient outcome [4-10]. Several recent studies have demonstrated an association between pre-transplant complete response (CR) and outcome in MM patients receiving ASCT [11-13]; however, CR did not represent the effectiveness of the conditioning regimen for ASCT directly. Furthermore, recent studies have identified post- rather than pre-transplant CR as an important prognostic factor [14].

In the present study, we therefore evaluated the prognostic influence of the post-transplant day $+14(D+14)$ bone marrow plasma cell percent (BMPCp) as a reflection of the efficacy of the conditioning regimen on PFS and OS in newly diagnosed MM patients.

\section{METHODS}

\section{Patients}

We retrospectively analyzed MM patients who were initially above ISS stage II and chemosensitive (i.e., achieved at least a partial response) to induction therapy between February 2003 and January 2008. Thirty-nine patients (26 males and 13 females; median age, 57 years [range, 37 to 63]) with MM were treated with the same induction therapy (vincristine, adriamycin, and dexamethasone [VAD]) followed by single ASCT. All patients received stem cell support following melphalan therapy as a conditioning regimen.

\section{Treatment schedule}

Four, three-week cycles of induction therapy with VAD were performed. Peripheral blood stem cell collections (PBSCCs) were accumulated following the administration of high-dose cyclophosphamide and mobilized with granulocyte colony-stimulating factor (G-CSF). The patients also received $200 \mathrm{mg} / \mathrm{m}^{2}$ melphalan as a conditioning regimen. Autologous blood stem cells were infused on day o through a central venous catheter preceded by an intravenous injection of $50 \mathrm{mg}$ of pheniramine maleate and $125 \mathrm{mg}$ of methylprednisone. Post-transplant, the patients received $5 \mu \mathrm{g} / \mathrm{kg}$ G-CSF subcutaneously each day from post-transplant day +3 until engraftment. The patients received prophylactic ciprofloxacin, itraconazole, and acyclovir. Following high-dose ASCT, all patients were scheduled to receive 2 years of maintenance therapy with interferon ( $3 \mu \mathrm{g}$ on 3 occasions weekly) and prednisone (50 $\mathrm{mg}$ on alternate days) if the disease status did not show progression.

\section{Cytogenetic study}

All patients underwent a bone marrow biopsy and conventional cytogenetic analysis at diagnosis following 3 cycles of therapy with VAD and post-transplant D+14. The BMPCp was determined using both aspirate smears and histological samples. The review included an examination of each bone marrow (BM) slide to obtain an estimate of cellularity and the number and proportion of plasma cells (PCs) per field in histological sections. In addition, differential counts for PCs, lymphocytes, and histiocytes were performed on the smears. Del (13q) was identified by interphase fluorescence in situ hybridization (FISH) of BM samples at diagnosis as the chromosomal abnormality was associated with a short PFS and OS in previous studies [10].

\section{Response assessment of induction therapy}

A response assessment of the induction therapy was performed after 3 cycles of therapy according to International Myeloma Working Group criteria [15]. Briefly, CR was defined by negative immunofixation on serum and urine, $<5 \%$ plasma cells in the BM, and the disappearance of any soft-tissue plasmacytoma. If present at baseline; a very good partial response (VGPR) was defined as a reduction of $\geq 90 \%$ in the serum M-protein level and a urine M-protein level of $<100 \mathrm{mg}$ after 24 hours. A partial response (PR) was defined as a $\geq 50 \%$ reduction in serum $\mathrm{M}$ protein and a reduction in the 24hour M-protein level by $\geq 90 \%$ or to $<200 \mathrm{mg}$, and a $\geq$ $50 \%$ reduction in size of the soft-tissue plasmacytoma. Patients not meeting the criteria for CR, VGPR, PR, or progressive disease (PD) were defined as having stable disease (SD). PD was defined as the presence of at least one of the following conditions: an increase of $\geq 25 \%$ from baseline in the serum or urine M-protein level with an absolute increase of at least $0.5 \mathrm{~g} / \mathrm{dL}$ and $200 \mathrm{mg}$ after 24 hours, respectively.

\section{Statistical analysis}

All statistical analyses were performed using SPSS version 14.0 (SPSS Inc., Chicago, IL, USA). The Mann- 
Whitney $U$ test was used to compare those patients with a BMPC of $\geq 2 \%$ on post-transplant D+14 with those showing a BMPCp of $<2 \%$. The relationship between BMPCp after 3 cycles of VAD therapy and BMPCp at post-transplant $\mathrm{D}+14$ was determined by Spearman correlation analysis. PFS was measured from the start of treatment to the date of progression. OS was measured from the start of treatment to the date of death or last follow-up visit. PFS and OS were estimated using the Kaplan-Meier method and compared with these two groups using the log-rank test. Cox proportional hazard models were used for univariate and multivariate analyses to evaluate the predictive value of BMPCp at post-transplant $\mathrm{D}+14$ on PFS and OS compared to other predictive factors (e.g., BMPCp at diagnosis, CR after 3 cycles of induction therapy, del [13q] by FISH, and ISS stage III at diagnosis).

\section{RESULTS}

The patients' characteristics are shown in Table 1 . The median follow-up duration was 28.0 months. The median PFS and OS were 29.1 and 42.1 months, respectively. The ISS at diagnosis was stage II $(n=25)$ or III $(n=14)$. The types of M protein were $\operatorname{IgG}(\mathrm{n}=23), \operatorname{IgA}(\mathrm{n}=12)$, and others $(\mathrm{n}=4)$. The median BMPCp at diagnosis was 43.0\% (range, 11 to 57); chromosomal abnormalities were found by conventional cytogenetic analysis in 15 patients. Del (13q) was detected by FISH in 8 patients. Following induction therapy with $\mathrm{VAD}, 12$ patients achieved a CR. The infused mean CD34+ stem cell dose was $4.1 \times 10^{6} / \mathrm{kg}$ (range, 2.1 to 6.1), while the median BMPCp at post-transplant $\mathrm{D}+14$ was $0.7 \%$ (range, o to 4.0). An analysis of the different cut-off levels between the 25 and $75 \%$ quartiles (range, 0.2 to 2.2) using the log-rank test determined that a BMPCp of $2 \%$ was the cut-off point yielding the greatest difference in PFS and OS; thus, this value was used as the cut-off level in our statistical analyses.

\section{Comparison of patient characteristics according to BMPCp at post-transplant $D+14$}

The baseline characteristics (age, sex, type, chromosomal abnormality, BMPCp at diagnosis, and stage) were compatible between the BMPCp $\geq 2 \%$ at post-transplant $\mathrm{D}+14$ and BMPCp $<2 \%$ groups (Table 1 ). The response following 3 cycles of VAD therapy was not different between the two BMPCp groups.

\section{Table 1. Baseline patient characteristics}

\begin{tabular}{|c|c|c|c|c|}
\hline Characteristics & Total & $\begin{array}{c}\mathrm{BMPC} \geq \geq 2 \% \\
\text { at post-transplant } \\
D+14\end{array}$ & $\begin{array}{c}\text { BMPCp }<2 \% \\
\text { at post-transplant } \\
\text { D+14 }\end{array}$ & $p$ value \\
\hline Age, yr & $57(37-63)$ & $52.5(37-60)$ & $57(38-63)$ & 0.210 \\
\hline Male/Female & $26 / 13$ & $7 / 5$ & $19 / 8$ & 0.558 \\
\hline \multicolumn{5}{|l|}{ Type } \\
\hline $\lg G$ & $23(59.0)$ & $7(18.0)$ & $16(41.0)$ & 0.954 \\
\hline $\lg A$ & $12(30.8)$ & $3(7.7)$ & $9(23.1)$ & 0.685 \\
\hline Others & $4(10.2)$ & $2(5.1)$ & $2(5.1)$ & 0.663 \\
\hline BMPCp at diagnosis, $\%$ & $43.0(11-57)$ & $47.5(11-57)$ & $38.0(23-56)$ & 0.599 \\
\hline Cytogenetic abnormality & $15(38.5)$ & $5(12.8)$ & $10(25.7)$ & 0.753 \\
\hline Del (13q) by FISH & $8(20.5)$ & $3(7.7)$ & $5(12.8)$ & \\
\hline Response after 3 cycle of VAD therapy & & & & 0.685 \\
\hline CR & $12(30.8)$ & $3(7.7)$ & $9(23.1)$ & \\
\hline Non-CR & $27(69.2)$ & $9(23.1)$ & $18(46.2)$ & \\
\hline ISS at diagnosis & & & & 0.327 \\
\hline$\|$ & $25(64.1)$ & $6(15.4)$ & $19(48.7)$ & \\
\hline III & $14(35.9)$ & $6(15.4)$ & $8(20.5)$ & \\
\hline CD34+ cell dose, x $10^{6} / \mathrm{kg}$, mean (range) & $4.1(2.1-6.1)$ & $4.1(2.1-5.4)$ & $4.0(2.6-6.1)$ & 0.391 \\
\hline
\end{tabular}

Values are presented as number (\%) or median (range) unless otherwise indicated.

BMPCp, bone marrow plasma cell percent; FISH, fluorescence in situ hybridization; VAD, vincristine, adriamycin, and dexamethasone; CR, complete response; ISS, international staging system. 


\section{Correlation between BMPCp after 3 cycles of VAD therapy and BMPCp on post-transplant $D+14$}

To estimate whether induction therapy with VAD influenced the post-transplant BMPCp, the correlation between BMPCps after 3 cycles of VAD therapy and posttransplant D+14 was estimated by Spearman correlation analysis. In the analysis, BMPCps between and above the two groups did not correlate $(r=-0.071, p=0.669$, Fig. 1$)$.

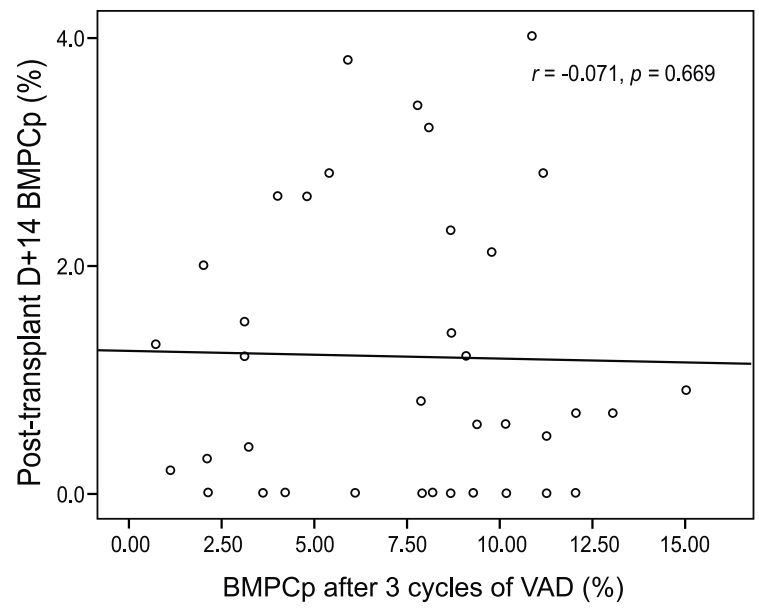

Figure 1. Correlation between BMPCp after 3 cycles of VAD and BMPCp at post-transplant D+14. The BMPCp following 3 cycles of VAD therapy did not correlate with the BMPCp at post-transplant $\mathrm{D}+14(r=-0.071, p=0.669)$. BMPCp, bone marrow plasma cell percent; $\mathrm{VAD}$, vincristine, adriamycin, and dexamethasone.

A

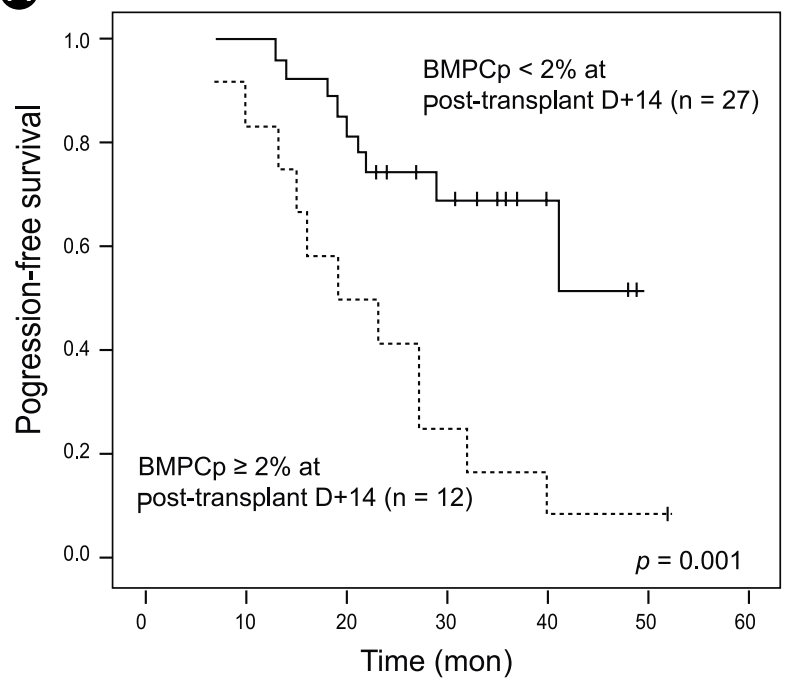

Impact of BMPCp at post-transplant D+14 on PFS and OS

The PFS and OS according to BMPCp at post-transplant $\mathrm{D}+14$ are shown in Fig. 2A and 2B, respectively. PFS in the BMPCp $\geq 2 \%$ group at post-transplant $\mathrm{D}+14$ was significantly shorter than in the $<2 \%$ group ( $p=0.001$, Fig. $2 A)$. Similarly, OS in the BMPC $\geq 2 \%$ group at post-transplant $\mathrm{D}+14$ was significantly shorter than in the BMPCp < $2 \%$ group ( $p=0.001$, Fig. $2 \mathrm{~B}$ ).

A univariate analysis revealed that BMPCp $\geq 2 \%$ at posttransplant D+14 was associated with a reduced PFS and OS (PFS, hazard ratio [HR] $=3.957,95 \%$ confidence interval $[\mathrm{CI}]=1.633-9.588, p=0.002$; OS, $\mathrm{HR}=4.048$, $95 \% \mathrm{CI}=1.622-10.103, p=0.003$, Table 2). Other factors (ISS stage III at diagnosis, $\geq 50 \%$ BMPCp at diagnosis, no CR after 3 cycles of VAD therapy, and del [13q] by FISH) were also correlated with the shorter PFS and OS (Table 2). A multivariate analysis using Cox proportional hazard models confirmed that a BMPCp $\geq 2 \%$ at post-transplant $\mathrm{D}+14$ and CR following 3 cycles of VAD therapy represented independent prognostic factors for PFS and OS (Table 3).
B

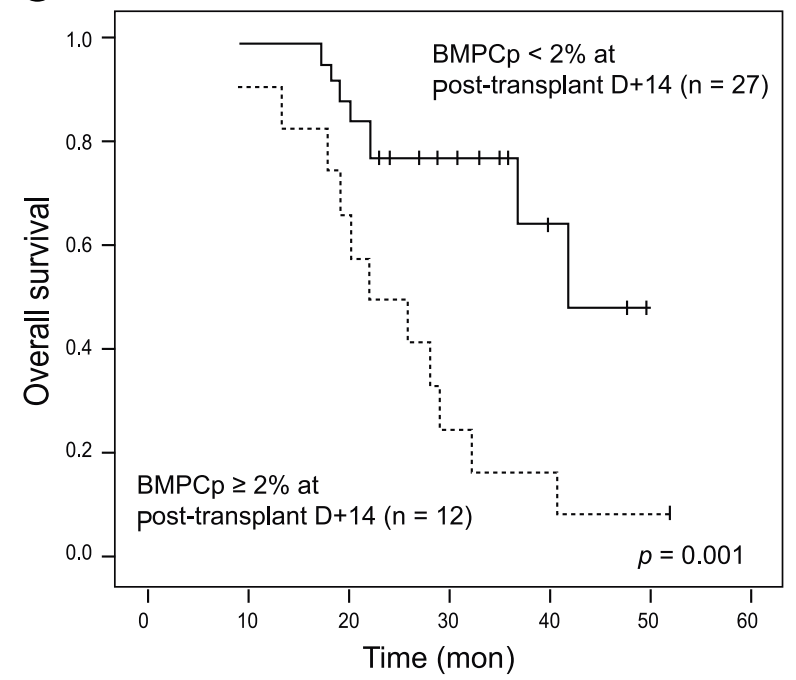

Figure 2. Influence of the BMPCp at post-transplant D+14 on (A) PFS and (B) OS. The patient group with a BMPCp $\geq 2 \%$ at post-transplant D+14 was associated with reductions in PFS and OS compared to the group with a BMPCp $<2 \%$ at post-transplant D+14 (PFS, $p=$ o.001; OS, $p$ = o.001). BMPCp, bone marrow plasma cell percent; PFS, progression-free survival; OS, overall survival. 
Table 2. Univariate analysis of prognostic factors for survival after ASCT

\begin{tabular}{|c|c|c|c|c|}
\hline \multirow[t]{2}{*}{ Prognostic factor } & \multicolumn{2}{|c|}{ Progression-free survival } & \multicolumn{2}{|c|}{ Overall survival } \\
\hline & $\mathrm{HR}(95 \% \mathrm{Cl})$ & $p$ value & $\mathrm{HR}(95 \% \mathrm{Cl})$ & $p$ value \\
\hline ISS stage III at diagnosis & $4.437(1.751-11.244)$ & 0.002 & $4.808(1.826-12.663)$ & 0.001 \\
\hline BMPCp at diagnosis $\geq 50 \%$ & $3.890(1.406-10.763)$ & 0.009 & $5.254(1.759-15.689)$ & 0.003 \\
\hline CR after 3 cycles of VAD therapy & $0.081(0.011-0.613)$ & 0.015 & $0.064(0.008-0.527)$ & 0.011 \\
\hline Del (13q) by FISH & $4.059(1.380-11.937)$ & 0.011 & $4.579(1.474-14.225)$ & 0.009 \\
\hline BMPCp $\geq 2 \%$ at post-transplant $D+14$ & $3.957(1.633-9.588)$ & 0.002 & $4.048(1.622-10.103)$ & 0.003 \\
\hline
\end{tabular}

ASCT, autologous stem cell transplantation; HR, hazard ratio; $\mathrm{Cl}$, confidence interval; ISS, international staging system; BMPCp, bone marrow plasma cell percent; $\mathrm{CR}$, complete response; VAD, vincristine, adriamycin, and dexamethasone; FISH, fluorescence in situ hybridization.

Table 3. Multivariate analysis of prognostic factors for survival after ASCT

\begin{tabular}{|c|c|c|c|c|}
\hline \multirow[t]{2}{*}{ Prognostic factor } & \multicolumn{2}{|c|}{ Progression-free survival } & \multicolumn{2}{|c|}{ Overall survival } \\
\hline & $\mathrm{HR}(95 \% \mathrm{Cl})$ & $p$ value & $\mathrm{HR}(95 \% \mathrm{Cl})$ & $p$ value \\
\hline ISS stage III at diagnosis & $2.224(0.789-6.268)$ & 0.131 & $2.231(0.750-6.635)$ & 0.149 \\
\hline BMPCp at diagnosis $\geq 50 \%$ & $0.997(0.287-3.468)$ & 0.996 & $1.377(0.352-5.377)$ & 0.646 \\
\hline CR after 3 cycles of VAD therapy & $0.072(0.009-0.583)$ & 0.014 & $0.055(0.005-0.562)$ & 0.015 \\
\hline Del (13q) by FISH & $3.171(0.936-10.749)$ & 0.064 & $2.557(0.787-8.312)$ & 0.119 \\
\hline BMPCp $\geq 2 \%$ at post-transplant $D+14$ & $4.426(1.470-13.327)$ & 0.008 & $3.545(1.071-11.727)$ & 0.038 \\
\hline
\end{tabular}

ASCT, autologous stem cell transplantation; HR, hazard ratio; $\mathrm{Cl}$, confidence interval; ISS, international staging system; BMPCp, bone marrow plasma cell percent; CR, complete response; VAD, vincristine, adriamycin, and dexamethasone; FISH, fluorescence in situ hybridization.

\section{DISCUSSION}

CR is defined by European Group for Blood and Marrow Transplant/International Myeloma Working Group uniform response criteria as the absence of serum and urine monoclonal $\mathrm{M}$ proteins by immunofixation (IFE) and $<5 \%$ PCs in the BM [15]. Several recent studies have shown that the pre-transplant CR is not associated with a better outcome in MM patients [11-13]. However, these studies did not interpret the importance of BMPC counts. Recent studies have demonstrated that the survival of patients who achieved a true CR (negative serum and urine IFE and $<5 \%$ BMPCs) was significantly longer than CR patients with $\geq 5 \%$ BMPCs [16]. This would be important to prevent the inclusion of a significant proportion of false-positive CR cases if the BM examination was removed. It is therefore suggested that the BMPC count is crucial for the prediction of survival in MM patients.

The present study showed that the post-transplant BMPCp was not associated with the BMPCp or response following 3 cycles of induction therapy. The BMPCp $\geq 2 \%$ at post-transplant $\mathrm{D}+14$ group correlated with a poor survival rate compared to the BMPCp $<2 \%$ group. The base- line characteristics, including the response after 3 cycles of VAD therapy, did not differ between the two groups. Furthermore, the BMPCp after 3 cycles of VAD therapy did not correlate with the post-transplant BMPCp. This result demonstrates that the efficacy of the conditioning regimen rather than the impact of induction therapy influences the post-transplant BMPCp. Although in previous studies the pre-transplant $\mathrm{CR}$ was not equivalent to the CR after 3 cycles of VAD therapy, the present study suggests that the post-transplant BMPCp represents a novel prognostic parameter irrespective of the pre-transplant disease status. Recent studies showing contrasting results have been reported, whereby the post-transplant CR rather than pre-transplant $\mathrm{CR}$ was associated with survival in newly diagnosed MM patients [14]. These studies suggest that achieving a response by high-dose ASCT could be an important prognostic factor of the outcome. Thus, the importance of the BMPCp could be understood in the context of post-transplant CR in these studies.

Other studies have revealed several prognostic factors such as cytogenetics, the plasma cell labeling index, ISS, and pre- and post-transplant CR status in ASCT, which may be useful in planning individualized treatment regimes [2-14]. Similarly, the present study shows that ISS 
stage III, a BMPCp $\geq 50 \%$ at diagnosis, CR after 3 cycles of VAD therapy, and del (13q) as well as a BMPCp $\geq 2 \%$ at post-transplant D+14 were associated with PFS and OS in a univariate analysis. However, our multivariate analysis revealed that only $\mathrm{CR}$ following 3 cycles of VAD therapy and BMPCp $\geq 2 \%$ at post-transplant D+14 were independent factors predicting PFS and OS. This early diagnostic approach at engraftment may have an important role in the prediction of outcome in ASCT.

Our data demonstrate that early detection of the residual myeloma burden by BM examination post-transplantation is an important factor in predicting the outcome for MM patients receiving single ASCT. A higher BMPCp at post-transplant D+14 may denote the necessity of the conditioning strategy, including high-intensity or novel agents, tandem ASCT, or more intensive maintenance therapy. Although this study investigated only a small number of patients and was retrospective in design, we identified a novel parameter for the early detection of the residual myeloma burden in ASCT. A well-designed prospective study that includes an examination of the BM on post-transplant $\mathrm{D}+14$ is needed to provide further information regarding these observations.

\section{Conflict of interest}

No potential conflict of interest relevant to this article was reported.

\section{REFERENCES}

1. Attal M, Harousseau JL, Stoppa AM, et al. A prospective, randomized trial of autologous bone marrow transplantation and chemotherapy in multiple myéloma. Intergroupe Français du Myélome. N Engl J Med 1996;335:91-97.

2. Child JA, Morgan GJ, Davies FE, et al. High-dose chemotherapy with hematopoietic stem-cell rescue for multiple myeloma. $\mathrm{N}$ Engl J Med 2003;348:1875-1883.

3. Bjorkstrand B, Svensson H, Goldschmidt H, et al. Alpha-interferon maintenance treatment is associated with improved survival after high-dose treatment and autologous stem cell transplantation in patients with multiple myeloma: a retrospective registry study from the European Group for Blood and Marrow Transplantation (EBMT). Bone Marrow Transplant 2001;27:511515
4. Stewart AK, Bergsagel PL, Greipp PR, et al. A practical guide to defining high-risk myeloma for clinical trials, patient counseling and choice of therapy. Leukemia 2007;21:529-534.

5. Avet-Loiseau H, Attal M, Moreau P, et al. Genetic abnormalities and survival in multiple myeloma: the experience of the Intergroupe Francophone du Myélome. Blood 2007;109:34893495 .

6. Greipp PR, San Miguel J, Durie BG, et al. International staging system for multiple myeloma. J Clin Oncol 2005;23:3412-3420.

7. Barlogie B, Shaughnessy J, Tricot G, et al. Treatment of multiple myeloma. Blood 2004;103:20-32.

8. Fonseca R, Blood E, Rue M, et al. Clinical and biologic implications of recurrent genomic aberrations in myeloma. Blood 2003;101:4569-4575

9. Rajkumar S, Fonseca R, Lacy M, et al. Abnormal cytogenetics predict poor survival after high-dose therapy and autologous blood cell transplantation in multiple myeloma. Bone Marrow Transplant 1999;24:497-503.

10. Worel N, Greinix H, Ackermann J, et al. Deletion of chromosome $13 q 14$ detected by fluorescence in situ hybridization has prognostic impact on survival after high-dose therapy in patients with multiple myeloma. Ann Hematol 2001;80:345-348.

11. Alexanian R, Weber D, Giralt S, et al. Impact of complete remission with intensive therapy in patients with responsive multiple myeloma. Bone Marrow Transplant 2001;27:1037-1043.

12. Dingli D, Pacheco JM, Nowakowski GS, et al. Relationship between depth of response and outcome in multiple myeloma. $\mathrm{J}$ Clin Oncol 2007;25:4933-4937.

13. Kim JS, Kim K, Cheong JW, et al. Complete remission status before autologous stem cell transplantation is an important prognostic factor in patients with multiple myeloma undergoing upfront single autologous transplantation. Biol Blood Marrow Transplant 2009;15:463-470.

14. Lahuerta JJ, Mateos MV, Martínez-López J, et al. Influence of pre- and post-transplantation responses on outcome of patients with multiple myeloma: sequential improvement of response and achievement of complete response are associated with longer survival. J Clin Oncol 2008;26:5775-5782.

15. Durie BG, Harousseau JL, Miguel JS, et al. International uniform response criteria for multiple myeloma. Leukemia 2006;20:14671473.

16. Chee CE, Kumar S, Larson DR, et al. The importance of bone marrow examination in determining complete response to therapy in patients with multiple myeloma. Blood 2009;114:26172618. 